\title{
BabyCare: apoio à decisão na atenção primária materno-infantil com computadores de mão
}

\author{
BabyCare: decision support system for primary child care \\ using personal digital assistant
}

\author{
Carmen Lúcia de Bartolo Costa ${ }^{1}$ \\ Vladimir Camelo Pinto ${ }^{1}$ \\ Orlando Lima Cardoso ${ }^{1}$ \\ M arcelo M assakazu Baba ${ }^{1}$ \\ Ivan Torres Pisa ${ }^{1}$ \\ Domingos Palma ${ }^{2}$ \\ Daniel Sigulem ${ }^{1}$
}

${ }^{1}$ Departamento de Informática em Saúde, UniversidadeFederal deSão Paulo. Rua Botucatu 862, Vila Clementino. 04023-062 São Paulo SP. carmenlbc@yahoo.com

${ }^{2}$ Departamento de Pediatria eN utrologia, Universidade Federal deSão Paulo.
Abstract Thepurpose of this work wasto develop a digital device - referred as BabyCare System for the collection, storage, and decision support for healthcare professionals and other concerned people, in order to assist patients in primary child carein deprived communities. Thissystem isbased on handheld devicetechnologies to be used locally in basic healthcare units in deprived communities, whether assisted or not by the Family H ealth Program/Strategy (PSF), as well as in ambulatory facilities and hospitals. Java was used as programming language. Evaluations have been conducted regarding 62 users at São Paulo city including volunteers from the Pastoral da Criança, an ecumenical institution for children. The applied questionnaires resulted in a high level of general acceptance (98.3\%); the on-site training was considered as appropriate (91.9\%); a perception of routine improvement and decrease in the time of consultation (100.0\%), and a decrease in the volume of paperwork ( $96.7 \%)$. The prototype has proven to be robust and effective for the use in deprived communities with precarious computer and telecommunication infrastructure.

Key words Clinical decision support systems, Public health care, Handheld computers, Primary health care
Resumo 0 objetivo desta pesquisa foi desenvolver um instrumento digital - aqui denominado Sistema BabyCare - para coleta, armazenamento e apoio à decisão dos profissionais de saúde e de mais envolvidos na assistência primária infantil em comunidades carentes. Esse sistema baseia-se em tecnologias de dispositivos móveis para utilização local em unidades básicas de saúde em comunidades carentes, assistidos ou não pelo Programa/Estratégia Saúde da Família (PSF), organizações não governamentais, ambulatóriosehospitais. U tilizou-se Java como linguagem de programação. Foram realizadas avaliações sobre 0 uso do sistema na cidade de São Paulo, envolvendo 62 usuários com diferentes formações, incluindo voluntários da Pastoral da Criança e do PSF. Os questionários aplicados resultam em alto índice de aceitação geral $(98,3 \%)$; treinamento in loco considerado adequado (91,9\%) ); percepção na melhoria na rotina ena redução detempo da consulta $(100,0 \%)$ e na redução no volume de documentos (96,7\%). Conclusão: o protóti po apresentou-se robusto e efici ente para uso em comunidades carentes com infraestrutura precária de informática e telecomunicação.

Palavras-chave Sistemas de apoio a decisões clínicas, Saúde pública, Computadoresdemão, Cuidados primários de saúde 


\section{Introdução}

Diariamente, 30 mil crianças morrem acometidas por doenças passíveis de serem prevenidas, como desnutrição, doenças respiratórias e doenças por falta de vacinas, seguindo-se a violência que se encontra em segundo lugar ${ }^{1,2}$. Dez miIhões de vidas poderiam ser salvas nos próximos dez anos se houvesse recursos para vacinação nos países pobres, segundo especialistas ${ }^{3}$. No Brasil, 27 milhões de crianças vivem abaixo da linha da pobreza, em moradias precárias como favelas, e fazem parte de famílias que têm renda mensal de até meio salário mínimo ${ }^{2}$. Aproximadamente $33,5 \%$ de brasileiros vivem nessas condições econômicas, e destes $45 \%$ são crianças que têm três vezes mais possibilidade de morrer antes dos cinco anos. 0 índice atual de desnutrição é de $19 \%$ entre os moradores de favelas, ante $5 \%$ entre os moradores urbanos, devido à falta de infraestrutura básica ${ }^{4}$.

M uitas são as causas que concorrem para esse quadro ocasionado principalmente pela pobreza e pelas desigualdades sociais $\varsigma^{5}$. D entre as medidas que poderiam ser tomadas para combatê-lo, destacam-se uma melhor equidade entre as regiões, saneamento básico, o combate ao baixo peso ao nascer (BPN) e da desnutrição, das doenças respiratórias, diarreicas, infectocontagiosas e parasitárias. A intensificação das campanhas de vacinação, melhoria da qualidade do pré natal, da escolaridade materna e ações educativas de prevenção à saúde, planejamento familiar e monitoramento de outros fatores de risco também são relevantes. As proporções continentais do país tornam ainda mais difíceiso estabelecimento de estratégi as e o cumprimento de metas para inclusão da população carente em programas assistenciais para atingir áreas geográficas e grupos populacionais mais vulneráveis e obter reduções significativas na mortalidade infantil. 0 governo e organizações não governamentais (ONGs) têm investido nosúltimos anosem programas de vacinação em massa e combate à desnutrição, alcançando altos índices de cobertura vacinal $^{2}$. Em várias regiões com pouco ou nenhum acesso do Programa de Agentes Comunitários de Saúde (Pacs) e Programa/Estratégia Saúde da Família (PSF), a ação conjunta ou isolada de diversas ONGs tem contribuído para o declínio desse quadro e a inclusão dessa população carenteem programas de informação eapoio à família. Entretanto, sem a criação denovas ações de políticas públicas e o investimento em sistemas de informação em saúde, considera-se difí- cil alcançar um resultado satisfatório que seja uniforme em todo o país.

Sabe-se que o uso de sistemas computadorizados auxilia, agiliza e garante mel hor qualidade no atendimento médico ao paciente ${ }^{6}$. Experiências $^{7,8}$ apontam para as vantagens do uso desses dispositivos para melhorar a qualidade da saúde, melhorando o processo da consulta e possibilitando acesso rápido aos dados dos pacientes. Recentemente, a Organização M undial da Saúde (OMS) destacou a grande demanda pelo desenvolvimento de sistemas de informação para a área de saúde 3 . Apontou também a necessidade de utilizar equipamentos mais modernos e versáteis capazes de serem transportados facilmente para áreas remotas. Assim, a mobilidade se apresenta como um fator essencial para se ampliar o sucesso do uso de sistemas de informação em saúde. Atualmente, observa-se ${ }^{9}$ um crescente número de artigos científicos e de aplicações na área de saúde utilizando personal digital assistance (PDA), também conhecido como computador de mão ou handheld. Entretanto, o número deaplicações em saúde utilizando PDA para apoio aos cuidados primários infantis ainda é escasso e demonstra que há muito campo para pesquisa nessa área ${ }^{10}$. Estudos conduzidos comparando o processo manual com o informatizado demonstraram que o processo manual émenos eficaz se comparado com os que utilizam $\mathrm{PDA}^{6}$. $\mathrm{Na}^{\text {Índia }}{ }^{11}$, Honduras e U ganda ${ }^{12}$, os PDAs vêm sendo empregados em aplicações de apoio ao diagnóstico e reduziram substancialmente 0 tempo gasto na consulta pelos profissionais de saúde, contribuindo deforma relevante para promover a qualidade do atendimento ao paciente.

São muitas as razões para a adoção de sistemas informatizados utilizando-se PDA, como maior facilidade no transporte do equipamento e a possibilidade de transmissão imediata da informação $0^{2,13}$. O monitoramento de crianças na faixa de 0-6 anos, especialmente as de baixa ren$\mathrm{da}^{14}$, seria um dos grandes objetivos que justificariam o uso desses equipamentos em programas de saúde. Estudos ${ }^{15-17}$ mostram que, quando identificamos que uma criança está desnutrida e a submetemos a programas de atenção básica para recuperação, ela apresenta melhoras significativas. Tal fato aponta que intervenções precoces em populações de risco auxiliam na diminuição da taxa de mortalidade infantil.

No Brasil temos cerca de 6 milhões de crianças para serem monitoradas e que se encontram em condições de severa degradação das condições humanas básicas, incluindo alimentação, 
água limpa, condições sanitárias, saúde, habitação, educação e informação². Em vista dessequadro precário, tem crescido o número deentidades evoluntários leigosquevêm auxiliando no cuidado à saúde. Esses voluntários muitas vezes identificam problemas de saúde menos graves, encaminhando os doentes àsunidades básicas deatendimento à saúde ${ }^{18}$. Visando dar 0 apoio a esses voluntárioseampliar seu âmbito deatuação, surge a necessidade de se oferecerem instrumentos informatizados para treinar e expandir o número de voluntários, bem como de se facilitar a interpretação dos dados coletados, para possivelmente melhorar as condições da assistência da saúde. 0 objetivo desse tipo de sistema de informação não é de fornecer um diagnóstico médico, mas sim de atuar como uma ferramenta de apoio, indicando a necessidade de encaminhamento do pacientea um procedimento médico. Ações como as citadas trariam um grande incremento no âmbito da atuação no PSF. Os voluntários, uma vez treinados, agiriam como intermediários, facilitando e promovendo a interação constante com as famílias, monitorando as condições de saúde como desnutrição, doenças respiratórias, atuando em campanhas de vacinação e encaminhando doentes aos órgãos competentes.

Considerando esse cenário, foi desenvolvido por Carmen Lúcia de Bartolo Costa um projeto de mestrado no Programa de Pós-Graduação em Informática em Saúde, do Departamento de Informática em Saúde (DIS), Universidade Federal de São Paulo (Unifesp), um protótipo baseado em computador de mão chamado Sistema BabyCare, para coleta e apoio à decisão na atenção primária materno-infantil. Esse protótipo oferece a possibilidade de coletar, armazenar e correlacionar informações de visitas anteriores do paciente, gráficos de crescimento, acompanhamento imunológico, histórico de saúde ocorrido no mês de referência e no ato da consulta para apoio aos profissionais de saúde objetivando trazer melhora na qualidade do atendimento. Esteartigo apresenta aspectos do desenvolvimento do Sistema BabyCare e os resultados da avaliação efetuada.

Este trabalho de pesquisa partiu da hipótese de que sistemas de apoio à decisão aplicados à atenção primária, baseados em computadores de mão, podem auxiliar na coletae no armazenamento dos dados relacionados, permitindo ainda a emissão de al ertas contendo procedimentos a serem adotados, com a possibilidade de instruções à distância aos profissionais promovendo uma educação continuada, cálculos para apoio aos profissionais de saúde e ajuda nas ações de políti- cas públicas pelos gestores. Assim, os resultados deste trabalho contribuem para a discussão na área sobreo aumento da aceitação do uso de tecnologia da informação e comunicação para auxiliar no apoio à atenção primária - em particular a infantil -, considerada como alta prioridade pelo governo federal, como se pode notar pelas ações do Projeto Nacional de Telemedicina para Atenção Básica (PNTAB), conduzido pelo M inistério da Saúde, universidades e órgãos executivos.

\section{M ateriais e métodos}

Foram realizadas observações na rotina das equipes do PSF, assim como na de voluntários leigos que atuam na prevenção e educação em saúde, mas não diagnosticam os pacientes. Quando necessário, os voluntários atuam como um elo entre as comunidades carentes e os órgãos de saúde. Esses grupos são constituídos de voluntários de ONGs internacionais (Compassion, Visão Mundial) e nacionais (ONG Pastoral da Criança), em que se observou a atuação dos voluntários de diversas comunidades.

Para a seleção dos campos que compõem a interface do programa, utilizaram-seformulários oficiais dos órgãos que foram acompanhados, além dos formulários da Organização Pan-Americana da Saúde (Opas) e dos questionários das ONGs, entre eles: PSF-Qualis e PSF/Pacs do Jardim M itsutami; Caderno do Líder ${ }^{18}$, guias eapostilas de capacitação da Pastoral da Criança; cartão da criança, fornecido pelo governo federal, e cartão da pastoral; livro e fichas da O pas/CLAP/ OMS sobre atenção materno-infantil; prontuário eletrônico do Instituto da Criança; prontuário el etrônico de pediatria do Setor deN utrologia da Unifesp. Foram consultados pediatras especialistas em imunologia, saúde pública, distúrbios em metabolismo e nutrição, e pediatra generalista para apoio ao desenvolvimento.

Para a eleição dos atributos dos campos da interface gráfica, analisaram-se todos os formulários, o processo de trabalho dos profissionais que coletam as informações e a melhor maneira de interação deles com o computador (técnicas de IHC). Foram verificados os seguintes aspectos: se havia redundância de informações nos documentos, as dificuldades e morosidade no preenchimento dos formulários, pontos que poderiam causar má interpretação, a introdução de campos não existentes no formulário, e como 0 processo poderia ser agilizado visando propor uma reordenação do processo de trabalho dos 
profissionais mencionados utilizando PDA. Com base nas tendências da literatura médica e de engenharia de sistemas, com o devido respaldo dos especialistas efetivamenteconsultados, na relevância da informação aos serviços prestados, e por consenso entre os especialistas consultados, foi efetivada a escolha dos campos na ordem em que os especialistas consideraram mais adequadas. Para a construção do software foram utilizados métodos de engenharia de software derivados da Extreme Programming (XP). Tendo em vista a portabilidade da aplicação para diversas plataformas, adotou-se o uso da linguagem Java. A portabilidade do protótipo, tamanho da memória e o uso de software livre foram considerados na definição da plataforma adotada. Como ambiente de desenvolvimento utilizou-se SuperWabaSDK 4.21a; EclipseJava Development Tools 2.1.3 (GNU) e Java 2 SDK Standard Edition 1.4.2.

Para desenvolvimento, testes etreinamento foram utilizados computadores de mão de diversos fornecedores e recursos diferenciados, para que os usuários pudessem se familiarizar com as tecnologias existentes e para que a equipe de desenvolvimento pudesse avaliar o desempenho em diferentes equipamentos. Entre os equipamentos, destacam-se: Partner GP200, da Gradiente, Compaq H P iPAQ H3970, I-mate Pocket PC, PalmOne Zire 72, PalmOne M 130, PalmOne Tungsten T2. Para avaliação estatística utilizou-semétodo deAltman et al. ${ }^{19}$, o software SPSS versão 10.1, recursos do pacote M icrosoft Office e o software Program Confidence Interval Analysis (CIA) versão 20.0 de Trevor Bryant, da University of Southampton.

Para a avaliação do protótipo BabyCare foram selecionados aleatoriamente 62 voluntários, de ambos os sexos, com idades variando entre 16 e 64 anos, com atuação em serviços de saúde pública. Dentre os voluntários incluem-sepediatras, enfermeiros, auxiliares de enfermagem, voluntários leigos de ONGs e equipe do PSF, especialmente agentes comunitários de saúde.

A avaliação do projeto baseou-se na aplicação de questionários pré-teste e pós-teste e pesquisa de satisfação sobre o protótipo, divididos em quatro fases.

A fase 1 teve como objetivo avaliar a usabilidade do sistema e o perfil do usuário por meio de um questionário de préteste com dez questões de múltipla escolha. Foi disponibilizado um tempo de 15 minutos para preenchimento do questionário, termo de voluntário e autorização de uso da sua imagem.

$\mathrm{Na}$ fase 2 houve um treinamento básico com turmas de no máximo seis participantes visando proporcionar condições de o usuário conhecer os recursos básicos de um computador de mão. Houve oportunidade para que os usuários esclarecessem suas dúvidas sobre a operação do equipamento em questão. Foram fornecidas explicações sobre os recursos e utilização do sistema BabyCare. Para cada voluntário foi disponibilizado um computador de mão por um período de noventa minutos.

$\mathrm{Na}$ fase 3 foi proposta a realização de uma tarefa para ser efetuada em trinta minutos visando observar-se o grau de interação e dificuldades dos usuários. 0 objetivo foi avaliar se 0 usuário conseguia acionar o sistema, incluir os dados referentes a uma criança, alterar os dados no caso de erro na digitação, ler os alertas, confirmar a inclusão do registro, consultar a existência de uma criança no cadastro e sair do programa.

A fase 4 teve como objetivo aplicar um questionário, com nove questões de múltipla escolha e uma questão livre, para avaliar os níveis de satisfação dos usuários em relação ao aplicativo, estimulando os usuários a escreverem, em campo texto livre, suas impressões, expectativas, opiniões e sugestões diversas sobre o processo proposto.

\section{Resultados}

Como primeiro resultado, foi desenvolvido o protótipo do sistema visando fornecer apoio a decisões de profissionais de saúde que trabalham na atenção primária materno-infantil de diferentes áreas de atuação. Em seguida, são apresentados os resultados dos questionários aplicados para avaliar o protótipo em um experimento de uso.

\section{Sistema BabyCare}

O sistema BabyCare propõe a aplicação de um modelo padronizado de perguntas para agilizar o trabal ho e ajudar as equipes de saúde na identificação de pacientes com maior necessidade de atendimento de forma rápida através da análise de gráficos, alertas, histórico de pacientes, além de já fornecer os cálculos para avaliação antropométrica e esquema vacinal.

Inicialmente, a coleta dedadoséreal izada pelo profissional de saúdeatuantena comunidadepor meio de PDA no atendimento domiciliar, como também no Centro de Atendimento Comunitário por meio de PDA e computador pessoal. A partir dos dados coletados, estes são transmiti- 
dos usando uma conjunção de tecnologias, como conexão sem fio, conexão USB e conexão debanda larga, de acordo com os recursos tecnológicos disponíveis em cada comunidade. A transmissão ocorre para um repositório local, que pode ser um computador servidor, e também para um repositório central, mediante um website na Internet, ambos utilizando um sistema gerenciador de banco de dados. 0 repositório local tem papel importante para possibilitar a atualização dos demais PDAslocais independentemente da conexão de Internet com o repositório central.

A partir desses repositórios de dados, relatórios e alertas manuais e automatizados podem ser gerados para diferentes entidades, considerando diferentes visões (conjunto de dados), dependendo do objetivo e da autorização de acesso definidos para cada participante no processo. Para a construção e a avaliação do protótipo, foram utilizados PDAs e conexões USB e de banda larga. Os alertas e relatórios automatizados contemplam o modelo do Sistema BabyCare, no entanto, eles não foram implantados durante essa fase da pesquisa. Em relação à confidencialidade dos dados, foi utilizado para o protótipo avaliado o uso de login e senha para acesso ao sistema, por parte do profissional da saúde. U ma aplicação real, para ser implantada em larga escala, requer a aplicação de técnicas de criptografia e marcação de dados para incrementar o modelo de permissão de acesso. Quanto à segurança, o programa solicita ao usuário o login e a senha, ena transmissão dos dados o sistema utiliza uma biblioteca e faz uma criptografia simples (SSL). No entanto, os autores acreditam que técnicas amplamente usadas para criptografar dados, como outros recursos das conexões SSL, M D5 e tantas outras, devem ser implementadas, na comunicação tanto com o repositório local quanto com o repositório central, sem qualquer ônus significativo para o uso do sistema.

O Sistema BabyCare construído dentro da proposta divide-se basicamente em quatro módulos principais (conjuntos de dados), utilizando ao todo 25 telas, relativos às telas de cadastro da criança, dados do nascimento, doenças do nascimento e ocorrências no mês.

0 módulo 1 possibilita a coleta de dados demográficos, nascimento, exames obrigatórios ao nascer, filiação, antecedentes de saúde dos pais, composição e estruturação familiar, condição socioeconômica, condições ambientais, especialmente se reside em áreas de risco, e também registra se a família está incluída em programas de ajuda à população. Emite ainda dicas sobre educação em saúde e alertas para direcionamento do procedimento a ser seguido pelo usuário.

0 módulo 2, sobre acompanhamento mensal, possibilita acompanhar informações sobre a saúde da criança no mês de referência e o estado atual de saúde. A avaliação consta de averiguar e controlar a administração das vacinas de acordo com a fai xa etária, conforme calendário do Programa Nacional de Imunização (PNI). Possibilita também verificar o tipo de vacina, calcular o intervalo mínimo entre as doses de vacinas e a emissão de alerta se houver falha ou atraso na indicação sobre a administração de vacinas. Permite, igualmente, definir rotinas diferenciadas para controle de vacinas atrasadas em crianças menores de 7 anos e uma avaliação especial para aquelas acima desta faixa etária. Sobre a rotina de ocorrências de saúde, coleta dados sobre doenças que acometeram a criança no mês, estado de saúde atual, se houve necessidade de atendimento em unidades básicas de saúde, se foram atendidas devidamente, se houve hospitalizações e o período. Possibilita a emissão de alertas para encaminhamento aos órgãos de saúde quando necessário ou de dicas de educação em saúde a serem apontadas aos pais, além de mostrar um histórico das visitas, contendo os problemasapresentados para posterior avaliação.

0 módulo 3, de acompanhamento psicomotor, visa coletar dados para avaliar o desenvolvimento emocional e psicológico e o desenvolvimento motor de acordo com a faixa etária. Incluem-se nesse módulo indicadores para registrar o raciocínio e reflexos inerentes à idade.

0 módulo 4, sobre a rotina de acompanhamento nutricional, representa uma ferramenta de apoio ao diagnóstico quanto às condições alimentares (dieta atual) e avaliação do estado nutricional. Com esses dados, o módulo possibilita avaliar se a criança se encontra desnutrida, obesa, com sobrepeso, baixa estatura e o grau de comprometimento. Foram adotados os critérios de Gómez (para crianças menores de 2 anos), critério deWaterlow (crianças maiores de 2 anos) e o critério Z-score da Organização Mundial da Saúde (OMS) para avaliação populacional em todas as idades.

\section{Avaliação com voluntários}

Do grupo dos 62 voluntários que participaram da avaliação, 83,9\% pertencem ao gênero feminino e $16,1 \%$ ao gênero masculino, com nível de escolaridade variando entre $2^{\circ}$ grau (29,5\%) 
e pós-graduação (34,4\%). Os usuários voluntários da Pastoral da Criança representaram $25,8 \%$, sendo os demais oriundos das áreas de nutrição $(22,6 \%)$, saúde $(21,0 \%)$, seguindo-se a área mé dica $(9,7 \%)$, enfermagem $(4,8 \%)$ e outras. Cabe destacar que uma grande parte dos usuários do sistema tem experiência de trabalho de mais de seis meses na área da saúde pública (70,7\%).

Os resultados dos questionários de pré-testes mostraram que muitos dos voluntários já faziam uso frequente de um computador pessoal $(46,8 \%)$, porém não o utilizavam como ferramenta de trabalho. Quando a avaliação deteve se nos conhecimentos sobre computadores de mão, $83,9 \%$ desconheciam ou nunca havia utilizado; apenas $16,1 \%$ responderam já dispor de conhecimento sobre 0 assunto e alegaram ter utilizado PDA ao menos uma vez.

As respostas do questionário pós-teste mostraram, por um lado, que cinco pessoas $(8,1 \%)$ não consideraram o treinamento adequado. Por outro lado, 57 usuários (91,9\%) conseguiram acompanhar bem o treinamento, independente mente do grau de escolaridade. Dos voluntários, $64,5 \%$ não encontraram dificuldades no uso do PDA, enquanto $35,5 \%$ dos usuários relataram ter tido al guma dificuldade.

O questionário de avaliação de satisfação quanto ao uso do protótipo mostrou que, durante a tarefa, 98,3\% reportaram seu uso altamente satisfatório. 0 número de voluntários que se mostraram resistentes ao uso de PDA não foi significante dentro da amostragem. Foram compiladas as expectativas apontadas pelos voluntários para a implantação e utilização real do Sistema BabyCare na área de saúde, obtendo os se guintes principais resultados: sobre a influência que o computador pessoal tem no trabalho que desempenham, 82,8\% apontaram que essa influência émuito importante; sobre o grau demelhoria que o Sistema BabyCare pode trazer na execução diária de tarefas, 95,1\% apontaram que houve muita melhoria; em relação ao módulo de cálculos, $98,4 \%$ apontaram que ele ajuda muito na redução do tempo da avaliação diagnóstica; sobre o potencial de redução no volume de documentos eno tempo na real ização da tarefa, 93,3\% informaram que houve muita redução; sobre 0 grau de dificuldade para incluir novas informações, $90 \%$ apontaram que houve nenhuma ou poucadificuldade; sobreo grau de dificuldadepara pesquisar informações, 91,9\% apontaram que houve nenhuma ou pouca dificuldade.

\section{Discussão}

A observação conduzida durante a construção do protótipo do Sistema BabyCare mostrou que o processo de atenção primária nas comunidades carentes avaliadas é precário. Ele é efetuado normalmente de forma manual, utilizando textos livres ou recursos tais como questionários preelaborados para coleta dos dados da criança, de sua família e do seu habitat. 0 atendimento é feito pelas equipes do Pacs, do PSF, médicos, enfermeiros, assim como por voluntários deONGs. Dependendo do órgão, 0 atendimento éfeito em postos de atendimento de saúde ou centros comunitários e/ou em domicílio. N esses processos manuais ocorrem muitos erros por escassez de informações, falta de padronização, resistência ou dificuldade de alguns usuários de seguir o padrão adotado, longas distâncias a serem percorridas atéo posto central deatendimento, onde ficam os especialistas ${ }^{20}$. Ainda há uma dificuldade de se manter um histórico das visitas passadas com dados para apoio à decisão principalmente em emergências, quando é comum ser 0 ACS ou voluntário a tomar uma decisão rápida.

Também há desconforto pelo acúmulo de documentos levados pelos profissionais de saúde até os locais de atendimento e dificuldades de se acharem as informações necessárias nos documentos rapidamente, para se ter mais eficácia no atendimento. Há também morosidade no preenchimento eenvio de notificação compulsória e no envio dos dados coletados aos gestores de saúde para promoção de políticas públicas adequadas em benefício das comunidades. No caso de existirem vários gestores responsáveis pela comunidade, écomum queinformações não cheguem a todos, resultando em diferentes pontos de vista sobre uma ação a ser adotada. Em relação às ONGs com pouca infraestrutura, também deve-se ressaltar que algumas informações ficam restritas só a ela, não sendo levada aos órgãos competentes para avaliação epidemiológica e/ou para tomada de ações pertinentes.

0 processo de atenção primária preconizado pelo Sistema BabyCare possibilita identificar os principais fatores de risco ${ }^{21}$ e assistir a comunidade com uma ferramenta de apoio à decisão aos profissionais de saúde. 0 sistema pode ser usado em consultórios, clínicas e especialmente em regiões rurais. 0 Sistema BabyCare foi concebido para rodar em computadores pessoais em áreas urbanas e em computadores de mão quando em áreas rurais para auxiliar na coleta das informações e apoio à decisão. 
Visando ajudar na atenção primária, o processo definido inclui a coleta com consistência dos dados digitados, emissão dealertas edicas deencaminhamento aosórgãos desaúde ou dicas deeducação em saúde, além de processamento dos dados, especialmenteindicando as vacinas, efetuando os cálculos de antropometria e avaliando doenças. Possibilita a geração de relatórios em papel quetambém podem ser enviados eletronicamente, de maneira automatizada ou sob demanda. Os dados do prontuário podem ser compartilhados de forma controlada pelos órgãos responsáveis pela saúde daquela comunidade. Por fim, os alertas epidemiológicos podem ser emitidos automaticamente independentemente da ação humana, potencialmenteresultando em maior agilidadena assistência em saúde para os gestores.

Nesse sentido, o sistema BabyCare, por sua flexibilidadeeaplicabilidade, mostra-seideal para utilização em áreas urbanas e rurais, podendo ser também usado em conjunto com outros sistemas governamentais e atuando como importante instrumento para a erradicação das principais causas de mortes de crianças, contribuindo para uma democratização do atendimento médico na área de saúde em todo o país. Nas áreas rurais, podem ser incluídos recursos de teleme dicina para melhorar a cobertura de saúde e monitoramento. Ao programa podem ser criados novos módulos para avaliação de outras doenças, educação em saúde e alertas. A vantagem da coleta e da obtenção de resultados em tempo real, por exemplo no caso de dosagem de hemoglobina, pode diminuir o tempo para se ter

\section{Colaboradores}

CLB Costa, VC Pinto, OL Cardoso, M M Baba, IT Pisa, D Palma e D Sigulem participaram da concepção do trabalho, da análise e interpretação dos dados, de sua redação, da revisão crítica e da leitura e aprovação da versão final. uma resposta do exame para diagnóstico e prescrição adequada dos profissionais de saúde, assim como diminuir as filas e o tempo para se conseguir fazer um exame de sangue, que em alguns lugares pode ser atéde três meses de espera.

\section{Conclusão}

0 presente estudo concluiu com base nos relatos dos profissionais e voluntários participantes da pesquisa e nos resultados obtidos dos questionários de avaliação que o desenvolvimento do Sistema BabyCare de coleta earmazenamento de informações, acoplado a guias de conduta automatizados, é capaz de otimizar o atendimento dos profissionais de saúde, voluntários e agentes comunitários nas comunidades carentes. 0 sistema não édediagnóstico médico esim de apoio nas visitas, com vistas a assistir emonitorar continuamente 0 desenvolvimento de crianças na faixa etária de 0 a 10 anos.

0 protótipo construído mostrou-se eficaz na realização dos objetivos propostos, inclusive quando foi operado por usuários leigos, de baixa escolaridade e inexperientes no uso de computadores pessoais ou de mão. Os questionários aplicados resultam em alto índice de aceitação geral $(98,3 \%)$; treinamento in loco considerado adequado (91,9\%); percepção na melhoria na rotina e na redução de tempo da consulta (100,0\%) e na concordância sobre a redução no volume de documentos (96,7\%).

\section{Agradecimentos}

Os autores agradecem ao apoio deAmérico Tomé e M aurício Ruiz, da Intel do Brasil; da Palm One; à Coordenação de Aperfeiçoamento de Pessoal de Nível Superior, pela concessão de bolsa; a Hilton Fernandes e Célio Hira, do Laboratório de Sistemas I ntegrados da Universidade de São Paulo; à participação da Pastoral da Criança e do PSF e a todos os especialistas voluntários que colaboraram com o estudo. 


\section{Referências}

1. Organização Mundial da Saúde. OMS. Contribuição da atenção integrada às doenças prevalentes da infância (AIDIPI) para atingir as metas de desenvolvimento para o milênio. 44을 Conselho Diretor, 55a Sessão do Comitê Regional; 2003 set 22-26; Washington; 2003.

2. Poirier $M$. Crianças de até 6 anos. 0 direito à sobrevivência e ao desenvolvimento. Situação da Infância Brasileira 2006. [cerca de 7 p.]. Brasília: Unicef; 2005. [acessado 2006 set 19]. Disponível em: http://www.unicef.org/brazil/sib06h.htm

3. Delhi GAVI Board M eetings. Opportunities for global health initiatives in the health system action agenda. Doc A.5 GHIs and health systems, 6-7 December, 2005. p. 16. [acessado 2006 set 19]. Disponível em: www.gavialliance.org/resources/17brd_5_H ealth SystemsGHIs_6D ec2005.pdf

4. Brasil terá 55 milhões vivendo em favelas até 2020 . BBC Brasil, 16 jun 2006. [online]. [acessado 2006 set 19]. Disponível em: http://www.bbc.co.uk/ portuguese/reporterbbc/story/2006/06/060616_onu_ habitat_novo_is.shtml

5. Programa das $N$ ações Unidas para o Desenvolvimento (PNUD). Nações Unidas, 7 setembro 2005. [acesso 2006 set 19]. Disponível em: http://www.pnud.org.br/ pobreza_desigualdade/reportagens/index.php?id01= $1445 \&$ lay $=$ pde

6. Sigulem D. Um novo paradigma de aprendizado na prática médica da U nifesp/EPM [tese de livre-docência]. São Paulo: Universidade Federal de São Paulo; 1997. p. 177.

7. Chen ES, Mendonça EA, McKnight LK, Stetson PD, Lei J, Cimino JJ. PalmCIS: A wireless handheld application for satisfying clinician information needs. J Am M ed Inform Assoc 2004; 11(1):19-28.

8. Palermo TM, Valenzuela D, Stork PP. A randomized trial of electronic versus paper pain diaries in children: impact on compliance, accuracy, and acceptability. Pain 2004; 107(3):213-219.

9. Grasso MA, Yen MJ, Mintz ML. Survey of handheld computing among medical students. Comput M ethods Programs Biomed 2006; 82(3):196-202.

10. Kho A, Henderson LE, Dressler DD, Kripalani $S$. Use of handheld computers in medical education. Journal of General Internal Medicine 2006; 21:531.

11. Anantraman V, Mikkelsen $T$, Khilnani R, Kumar VS, Pentland A, Ohno-M achado L. Open source handheld-based EM R for paramedics working in rural areas. Proc AM IA Symp 2002; 1:12-6.

12. Waldick L. New wireless network for Uganda's healthcare workers. IDRC Reports [online]. [acessado 2006 set 19]. Disponível em: http://web.idrc.ca/en/ev47580-201-1-DO_TOPIC.html
13. Clayton C. ONG World Vision, Inglaterra, 2006 [online]. [acessado 2006 set 19]. Disponível em: http:/ /www.worldvision.org.uk

14. Lessa C, Devincenzi MU, Sigulem DM. Comparação da situação nutricional de crianças de baixa renda no segundo ano de vida, antes e após a implantação de programa de atenção primária à saúde. Cad Saude Publica 2003; 19(2):505-514.

15. Centro de Recuperação e Educação Nutricional. [online]. [citado 2006 maio 20] [acessado 2006 set 19]. Disponível em: http://www.unifesp.br/suplem/ cren

16. Fernandes BS, Fernandes MTB, Bismarck-Nasr EM, Albuquerque M P. Abordagem clínica e preventiva, livro 3. Solymos GMB, Sawaya $A L$, organizadores. Coleção Vencendo a Desnutrição. São Paulo: Salus Paulista; 2002. [acessado 2006 set 19]. Disponível em: http:/ /www.desnutricao.org.br/manuais/downloads/ Clinica.pdf

17. Sawaya SM. Abordagem pedagógica, livro 5. Solymos GM B, Sawaya AL, organizadores. Coleção Vencendo a Desnutrição. São Paulo: Salus Paulista; 2002. [acessado 2006 set 19]. Disponível em: http://www. desnutricao.org.br/manuais/downloads/Pedago.pdf

18. Guia do líder da Pastoral da Criança. Curitiba: Pastoral da Criança, Conferência Nacional dos Bispos do Brasil; 2000.

19. Altman DG, Bryant TN, Gardner MJ, Machin D, editors. Statistics with confidence: confidence intervals and statistical guidelines, 2nd edition. British M edical Journal. London; 2000.

20. Costa CLB. Sistema BabyCare: sistema de coleta e apoio à decisão na atenção primária materno infantil para comunidades carentes baseado em dispositivos móveis [dissertação]. São Paulo: Universidade Federal de São Paulo; 2006.

21. Palma D. Avaliação da condição nutricional. J Pediatr 1995; 71(3):125-126.

Artigo apresentado em 18/04/2007

Aprovado em 08/09/2007

Versão final apresentada em 21/11/2007 